\title{
Suivi des feux de brousse en Afrique de l'Ouest et au Sahel, un outil d'aide à la décision
}

\author{
Issa GARBA, Sanoussi ABDOU AMADOU*, Boubacar BARRY et \\ Souleymane OUEDRAOGO
}

Division Maitrise de l'Eau et Lutte Contre la Désertification/ Département Information et Recherche, Centre Régional Agrhymet, BP 11011 Niamey, Niger.

*Auteur correspondant ; E-mail : aasanoussi@hotmail.fr ; Tél. : (+227) 96052557

Received: 10-06-2021

Accepted: $15-10-2021$

Published: 31-12-2021

\section{RESUME}

Les feux de brousse sont courants en Afrique de l'Ouest et au Sahel. Ils contribuent à modifier sans cesse le paysage. Cette étude a été réalisée en Afrique de l'Ouest et en Mauritanie. Elle visait l'élaboration de plaidoyer pour la gestion des feux de brousse. Il s'agit plus spécifiquement, de contribuer à l'amélioration des connaissances sur la distribution spatiale et temporelle des feux de brousse. Pour cela, les données de MODIS (Moderate-Resolution Imaging Spectroradiometer) allant de 2014 à 2020 ont été utilisées. La répartition des feux actifs durant la saison 2019/2020 a été analysée et comparée à la moyenne des 5 dernières années précédant la période d'étude. De façon générale, la saison des feux démarre entre octobre à novembre et se termine en avril, avec des pics qui se manifestent à partir de décembre. Il ressort que $70 \%$ des occurrences des feux apparaissent entre décembre et février ce qui a permis de classer les pays en 7 groupes. Cette étude a également montré que le taux de détection des feux dépend du type d'occupation de sol. Enfin, elle montre l'importance des informations produites pour une meilleure gestion des ressources naturelles disponibles dans un contexte de changement climatique.

(C) 2021 International Formulae Group. All rights reserved.

Mots clés : Environnement, MODIS, feux actifs, distribution spatiale, distribution temporelle.

\section{Wildfires monitoring in West Africa and the Sahel, a decision support tool}

\begin{abstract}
Wildfires are common in West Africa and the Sahel and contributes to shape the landscape. The study was carried out in West Africa and Mauritania. The main goal of this study is a plea for monitoring wildfires in West Africa. The specific goal was to improve knowledge on the spatial and temporal distribution of wildfires in West Africa. Data from (2014-2020) of Moderate-Resolution Imaging Spectroradiometer (MODIS) have been utilized and processed using descriptive statistics. Repartition of active fire detection during 2019/2020 has been studied and compared with the mean of the previous five years (2014-2018). In general, fire season begins from October to November and ends in April with peaks starting in December. Our study revealed that $70 \%$ of wildfires are observed from December to February and this helps to classify the countries of the study area into 7 groups. Moreover, it has been showed that fire detection rate depends on the type of soil occupation. Finally,
\end{abstract}


this study shows that data from remote sensing can be used to provide information to help decision-making for monitoring the available natural resources under a climate change context.

(C) 2021 International Formulae Group. All rights reserved.

Keywords : Environment, MODIS, active fire, spatial distribution, temporal distribution.

\section{INTRODUCTION}

Qu'ils soient accidentels ou intentionnels, les feux de brousse font partie intégrante des facteurs déterminants qui contribuent à la dégradation des ressources naturelles en Afrique de l'Ouest. La nature de la végétation, du paysage, des facteurs climatiques et anthropiques font que l'occurrence des feux ainsi que l'étendue des surfaces brulées varient d'un pays à un autre, d'une région forestière à une savane boisée et/ou couverte de graminées (Valea et Ballouche, 2012). Dans ces zones, le feu survient pour diverses raisons. Dans certaines localités, il est allumé pour la chasse dans le but de rabattre le gibier ou d'enfumer les terriers ou les ruches (Kana et Etouna, 2006). Cela concerne surtout les aires protégées. Il peut aussi être allumé pour des raisons agricoles dans le but de défricher les champs. Le feu peut aussi être accidentel, lorsqu'aux abords des routes, il est jeté des cigarettes non encore éteintes, ou lorsqu'un feu de campement n'est complètement pas éteint, qu'il s'agisse des parcs ou des zones non protégées à végétation plus ou moins dense (Takahata et al., 2010).

Les effets que produisent les feux sur les ressources naturelles, notamment la végétation, le sol et la biodiversité varient selon les périodes. En effet, s'ils sont allumés juste à la fin de la saison pluvieuse, l'herbe n'est pas complètement sèche et le sol garde une certaine humidité par endroit. Ce taux d'humidité réduit l'efficacité des flammes et par là même l'étendue des surfaces brulées (Valea et Ballouche, 2012). Ce type de feux est dit précoce et caractérise les mois d'octobre et novembre en Afrique de l'Ouest. Il est également moins destructeur. En revanche, le feu déclenché pendant la période de janvier à avril est plus destructeur, produit de hautes flammes et est considéré comme dévastateur de la végétation et du sol (Doamba et al., 2014).
Ce feu concerne aussi souvent des étendues plus grandes.

Dans le sahel, zone d'élevage par excellence, souvent, la faible pluviométrie transforme l'effet bénéfique des feux en un désastre pour les éleveurs en emportant la quasi-totalité de la biomasse sèche nécessaire pour l'alimentation du bétail. Selon Ahouagan et al. (2010) le feu emporte $80 \%$ à $90 \%$ de la biomasse sèche après son passage. Il faut également noter que les feux de brousse constituent un fléau climatique parce qu'en brulant, la brousse dégage du dioxyde de carbone $\left(\mathrm{CO}_{2}\right)$ qui est l'un des principaux gaz à effet de serre (GIEC, 2014; Le et al., 2014). D'autres gaz à effet de serre comme le protoxyde d'azote $\left(\mathrm{N}_{2} \mathrm{O}\right)$ sont également libérés dans l'atmosphère de même que les particules de suie qui contribuent dans la pollution de la basse atmosphère conduisant ainsi à des problèmes de santé publique (Wang et al., 2010; Zhang, 2011; Lin et al., 2012; Price et al., 2012).

Depuis quelques années, les produits satellitaires peuvent être utilisés pour la détection des feux ou l'estimation des dégâts qu'ils causent. Aujourd'hui, la télédétection a réalisé des progrès énormes si bien que les feux de brousse peuvent être détectés avec une résolution temporelle assez importante (quasiment toute la journée) et une résolution spatiale de l'ordre du kilomètre voire même moins. Les feux de brousse posent des défis de plus en plus importants si bien que même les pays développés sont extrêmement préoccupés par leur fréquence ainsi que l'ampleur des dégâts matériels et parfois humains dont ils sont responsables. En effet, en 2018 dans l'ouest des Etats Unis, un feu a coûté la vie à une centaine de personnes et avait fait 24 milliards de dégât matériel (Kganyago et Shikwambana, 2020). 
Plusieurs études sur les feux de brousse ont été réalisées à travers le monde. L'effet des feux sur les sols, les indices de l'intensité des feux dans les savanes africaines, la dynamique des feux dans les aires protégées ont localement été étudiés. Des études sur les surfaces brulées ont également été réalisées en utilisant les produits satellitaires. Cependant, peu d'auteurs se sont penchés sur la répartition temporelle des feux de brousse à l'échelle régionale et son interaction avec l'occupation des sols.

Ainsi, au vu des dégâts multiples dont ils sont responsables, il apparait clairement que cataloguer les occurrences des feux de brousse permettra aux acteurs de l'environnement et ceux chargés de la gestion des aires protégées de procéder à un monitoring plus efficace des feux de brousse. L'objectif de cette étude était de contribuer à la protection de l'environnement ouest africain à travers l'élaboration des produits de plaidoyer pour la prise de décision dans la lutte contre les effets néfastes des feux de brousse. Il s'agissait plus spécifiquement de déterminer, en utilisant la télédétection, les périodes de début et de fin, la période de pic ainsi que la distribution spatiale des feux de brousse. Il sera également recherché des éventuelles variations interannuelles en procédant à une comparaison par rapport à une série de référence.

\section{MATERIEL ET METHODES}

\section{Présentation de la zone d'étude}

La zone d'étude regroupe les pays du Comité Inter-Etat de Lutte contre la Sécheresse au Sahel (CILSS) et de la Communauté Economique des Etats de l'Afrique de l'Ouest (CEDEAO) à l'exception du Cap Vert. Il s'agit du Bénin, Burkina Faso, Côte d'Ivoire, Gambie, Ghana, Guinée, Guinée Bissau, Libéria, Mali, Mauritanie, Niger, Nigéria, Sénégal, Sierra Leone et le Tchad (Figure 1). Le climat est du type tropical humide au niveau des pays côtiers à sahélien vers le nord. La pluviométrie varie en moyenne du sud au nord entre 2000 à $1500 \mathrm{~mm}$ dans le domaine soudanien, 600 à $150 \mathrm{~mm}$ au sahel (Hiernaux et Le Houérou, 2006; N'Dri et al., 2018). Les pays aux latitudes inférieures à $10{ }^{\circ} \mathrm{N}$ enregistrent pour certains, jusqu'à 4 saisons dont deux saisons de pluie et deux saisons sèches. Au-delà, l'année est marquée par deux saisons dont une courte saison de pluie allant de juin à septembre et une longue saison sèche allant d'octobre à mai. Notons qu'à partir des pays côtiers la pluviométrie diminue progressivement au fur et à mesure que la latitude augmente. Au-delà du $20^{\text {ème }}$ parallèle commence le désert du Sahara. Les températures sont plus élevées au niveau des pays sahéliens avec une moyenne annuelle de 28 à $30{ }^{\circ} \mathrm{C}$ et des maximas qui peuvent atteindre $45{ }^{\circ} \mathrm{C}$ pendant la période avril à juin (Hiernaux et Le Houérou, 2006). Cependant, au niveau des pays côtiers, les températures sont plus basses, conséquences d'un taux d'humidité plus élevé. Au Sahel, l'harmattan, vent chaud et sec de direction nord ou nord-est, souffle pendant la saison sèche. Durant une courte période (environ 3 mois), un vent venant de l'océan Atlantique de direction sud ou sudouest appelé mousson balaye la zone et transfert de l'humidité sur le continent qui se traduit par des pluies (Zeineddine, 2020).

Le paysage est caractérisé par la forêt dense à claire, la savane arborée et savane herbeuse dans le domaine soudanien (Cisse et al., 2020). Dans le domaine sahélien, elle est composée de savane arborée, arbustive et herbeuse en général.

\section{Données de l'étude}

Les données de la présente étude ont été obtenues auprès de NASA's Fire Information for Ressource Management System (FIRMS) à l'adresse (https://earthdata.nasa.gov/firms). Celles-ci proviennent du capteur MODIS (Moderate-Resolution Imaging Spectroradiometer) embarqué sur les satellites Terra et Aqua. Le satellite Terra parcourt la terre du nord au sud en passant par l'équateur dans la matinée alors que le satellite Aqua tourne autour de la terre du sud au nord en passant par l'équateur dans l'après-midi. Chaque feu actif est représenté par un point avec une résolution de $1 \mathrm{~km}$. Cette couverture permet la détection du maximum des feux qui ont lieu dans la journée. Ces données couvrent la période étudiée (2019/2020) ainsi que les 5 
dernières années (DA) de la période de référence (2014-2018) sur l'ensemble des pays de la zone d'étude. Une grille vectorielle de 10 $\mathrm{km}$ de côté a servi au calcul des densités de détection des foyers de feu. Une couche vectorielle des unités administratives des pays du CILSS et de la CEDEAO a également été utilisée.

\section{Méthodologie}

Les données de feux actifs des pays du CILSS et de la CEDEAO allant de 2014 à 2020 ont été analysées dans l'espace et dans le temps. A partir des fichiers shapefiles des feux actifs, une intersection spatiale avec la couche des limites administratives a permis de créer un champ qui permet de faire l'agrégation par unité administrative (Figure 2). Les champs année, mois et jour ont également été créés pour faciliter les agrégations temporelles. Ainsi des moyennes mensuelles ont été calculées sur la série. Celle-ci est représentée sous forme d'histogrammes qui offrent une meilleure appréciation des variations des occurrences des feux plutôt que les courbes. Par la suite elles ont été comparées à la moyenne de la série 2014-2018 dont les données ont été constituées de la même manière que pour la saison 2019/2020. En outre, les données sous format shapefile ont servi à l'analyse spatiale. La grille vectorielle de $10 \mathrm{~km}$ de côté a été utilisée pour calculer le nombre moyen de détection des foyers de feu pendant les 5 dernières saisons et celle en cours. Par la suite, une simple comparaison est faite entre les champs de densité moyenne de la saison 2019/2020 et celle 2014-2018 pour voir la distribution spatiale.

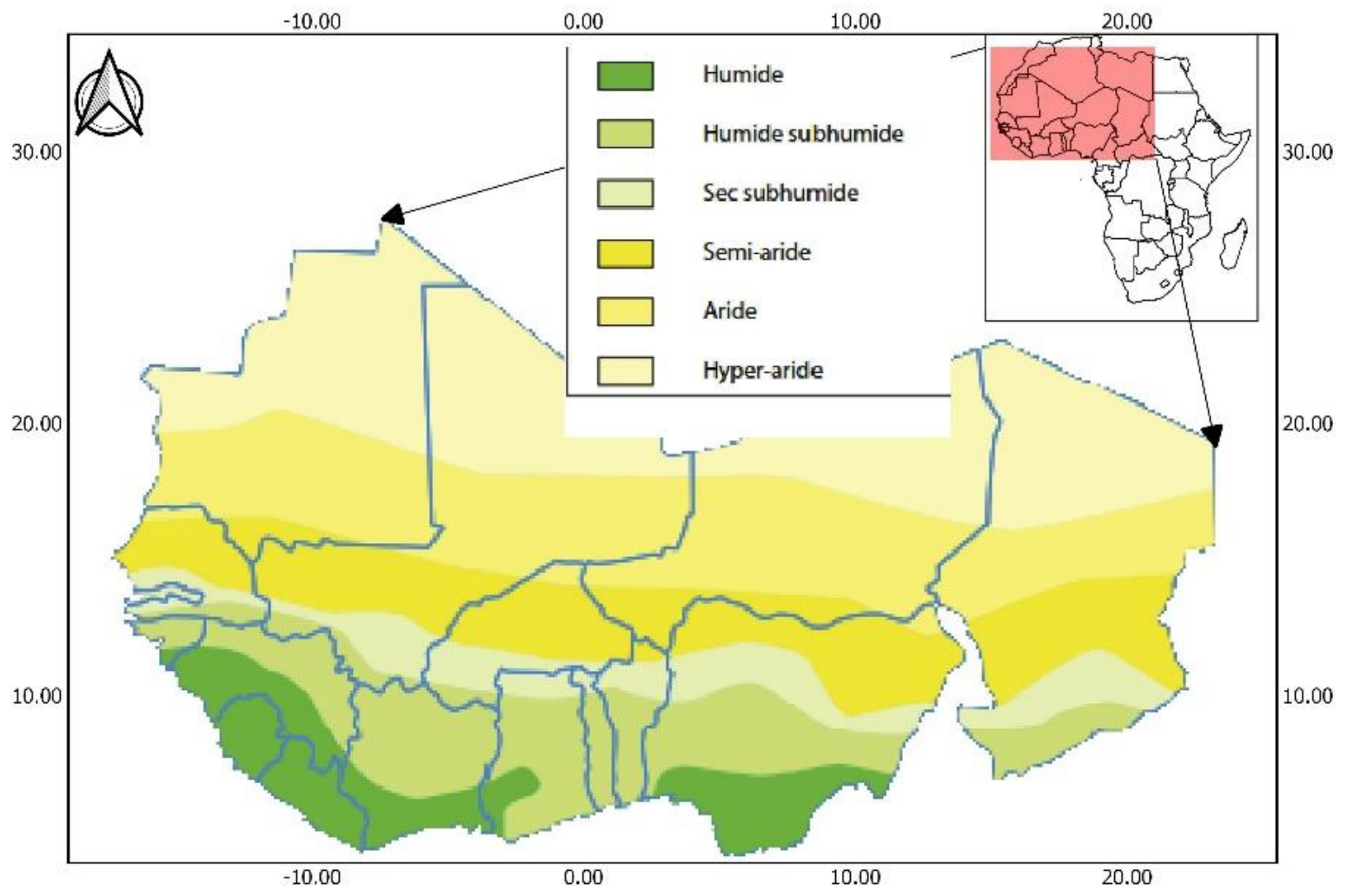

Figure 1 : Localisation de la zone étude et distribution spatiale des zones climatiques (d'après OMM et CSAO, (2009) Atlas régional de l'Afrique de l'Ouest). 


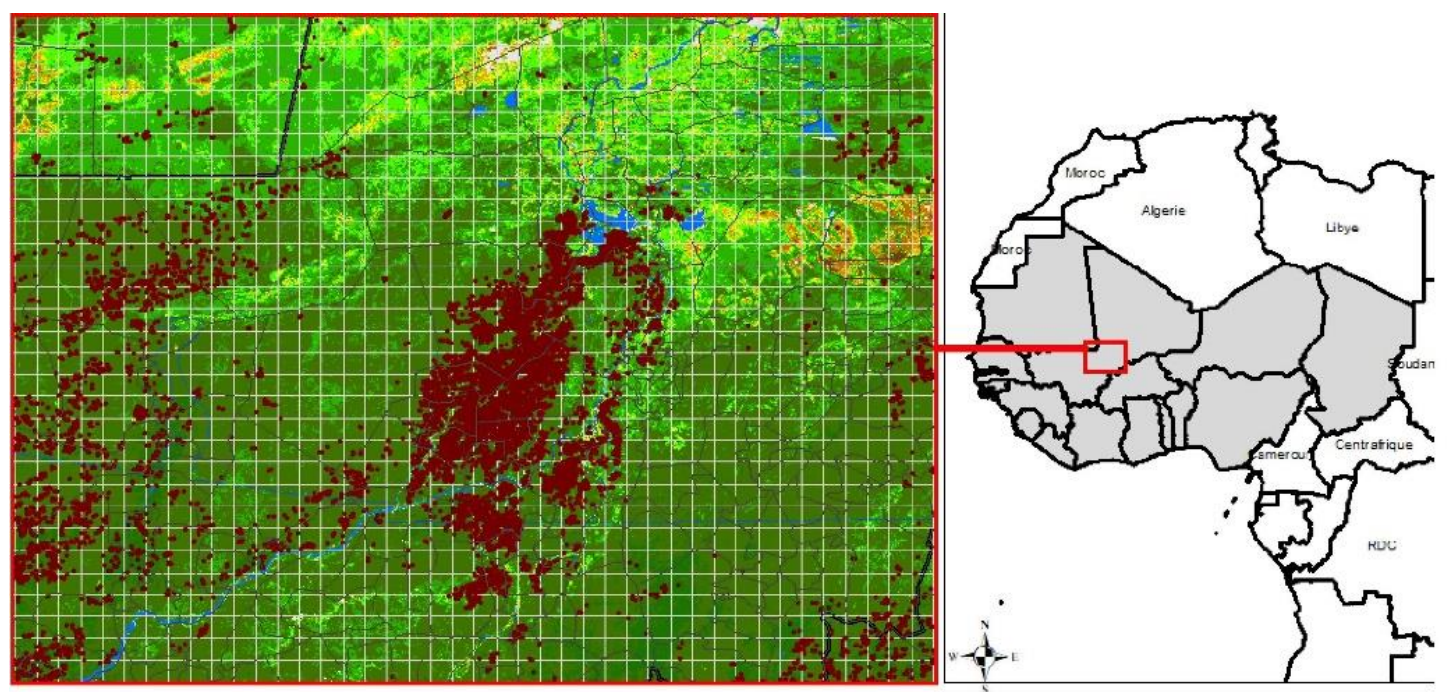

Production fourragere en kg.MS.ha-1 au 30 octobre 2020

\section{Feux actifs \\ Grile de $10 \mathrm{~km}$ de côté \\ Eau

 \\ RESULTATS \\ Observation générale et variabilité interannuelle}



$100-200$

$100-200$
$\square \quad 200-300$

$300-600$

$600-1200$

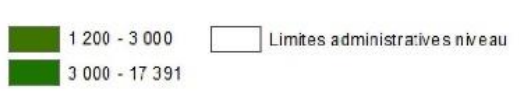

Figure 2 : Exemple de couplage des différentes couches.

Durant la saison 2019/2020, toute l'Afrique de l'Ouest a été soumise à des feux de brousse plus ou moins intenses depuis le Sénégal jusqu'au sud du Tchad (Figure 3). La Mauritanie, le nord Mali et du Tchad, ainsi que le Niger sont épargnés par les feux de brousse à l'exception du parc de W. D'ouest en est, on observe une concentration des occurrences des feux dans l'extrême ouest (Sénégal, Guinée, Sierra Leone), au centre (Ghana, Togo) et l'extrême sud du Tchad. Localement, la Côte d'Ivoire et le Nigeria ont également subi des feux à répétition.

La tendance des feux actifs au cours de la saison 2019/2020 dans l'ensemble des pays de l'Afrique de l'Ouest et du Sahel est à la baisse, de l'ordre de $2 \%$ par rapport à la moyenne dans des 5 dernières années (Figure 4). En effet, les occurrences de feux relevées dans les pays côtiers (Guinée, Togo, Côte d'Ivoire, Bénin, Libéria et Sierra Leone) restent inférieures par rapport cette moyenne. La répartition mensuelle des occurrences de feux montre que cette baisse globale cache d'importantes variabilités selon les zones bioclimatiques, les pays et les mois. Localement, des baisses sont observées dans le sud-est du Sénégal, à l'extrême ouest du Mali, en Guinée, le nord de la Sierra Leone, le nordest de la Côte d'Ivoire, la moitié nord du Ghana, une bonne partie du Togo, la zone du Parc de $\mathrm{W}$ des trois pays (Bénin, Burkina, Niger), le sud et l'extrême nord-est du Nigeria et le sud du Tchad.

L'examen de la répartition temporelle des feux indique que $70 \%$ des occurrences apparaissent entre décembre et février. Toutefois, les occurrences observées en février 2020 restent largement au-dessus de celles de la série (Figure 5). L'importance des occurrences dans les pays de l'Afrique de l'Ouest et leur temporalité permet de distinguer d'une part deux grandes zones :

- Les pays ayant une composante forestière importante que sont le Libéria, la Sierra Leone, la GuinéeBissau, la Gambie, la Guinée, la Côte d'Ivoire, le Nigéria, le Ghana, le Togo, le Bénin ;

- Les pays ayant une composante sahélienne plus importante que sont le 
Burkina Faso, le Niger, le Mali, la Mauritanie, le Sénégal et le Tchad.

Ainsi, l'ensemble des pays peuvent être classés en 7 groupes suivants :

- Groupe 1 (Le Libéria et la Sierra Leone) : ces deux pays présentent des occurrences de feux très importantes en avril. Leur caractéristique principale est que la saison des feux commence en décembre-janvier et se termine en avril. Les occurrences sont presque inexistantes en octobre et novembre ;

- Groupe 2 (La Guinée-Bissau, La Gambie et la Guinée) : ces trois pays présentent des occurrences de feux très importantes en février avec de faibles occurrences en octobre et novembre ;

- Groupe 3 (La Côte d'Ivoire, le Nigéria) : les occurrences des feux sont surtout concentrées en décembre, janvier et février ;

- Groupe 4 (Le Ghana, le Togo et le Bénin) : comme le groupe 3 , les occurrences sont centrées sur les mois de décembre à février, mais avec de fortes occurrences en décembre. Il est à noter que le Bénin enregistre des détections de feux actifs non négligeables en novembre;

- Groupe 5 (La Mauritanie, le Mali, le Sénégal) : le démarrage des occurrences de feu se situe en novembre avec un étalement sur les mois de décembre et janvier ;

- Groupe 6 (Le Tchad et le Burkina Faso) : les occurrences des feux s'étalent sur 4 mois, de novembre à février avec néanmoins de petites détections pendant le reste de la saison sèche ;

- Groupe 7 (Le Niger) : les occurrences des feux démarrent en octobre et s'étalent sur toute la saison sèche avec une forte densité en novembre suivi d'une baisse progressive de décembre à avril.
Analyse des variabilités intra-pays par rapport à la série de référence

Les pays du groupe 1

En Sierra Leone, le nombre d'occurrences de feux est en baisse de 19\% par rapport à la moyenne des 5 dernières années. La tendance de la saisonnalité a très peu varié par rapport à la moyenne des 5 dernières saisons avec une concentration des occurrences durant les 4 premiers mois de l'année. Les $62 \%$ des occurrences ont été enregistrés pendant les mois de mars et avril (Figure 6a). Au Libéria également, une baisse des occurrences de $18 \%$ est relevée avec une saisonnalité qui diffère très peu de celle de la moyenne des 5 dernières années. Les importantes occurrences de feux relevées en février, mars et avril restent quand même inférieures aux moyennes mensuelles (Figure 6b).

\section{Les pays du groupe 2}

En Gambie, une forte augmentation des occurrences des feux de l'ordre de $45 \%$ est observée par rapport à la moyenne des 5 dernières années. La saison des feux a démarré en novembre avec une très forte recrudescence en février. Les $38 \%$ des occurrences ont été observés au cours du seul mois de février qui a enregistré environ $90 \%$ plus de feu par rapport à la moyenne des 5 dernières années du même mois (Figure 6c). En Guinée Conakry par contre, une baisse de 7\% des occurrences a été enregistrée. La saison des feux a démarré véritablement en novembre et s'est poursuivie jusqu'en avril. Les plus importantes détections sont enregistrées durant les mois de décembre, janvier, févier. La variation mensuelle des occurrences de la saison 2019/2020 comparée à la moyenne indique que le mois de janvier a enregistré plus de feux. Les occurrences des mois de mars et d'avril sont inférieures aux moyennes des 5 dernières années (Figure 6d). En Guinée-Bissau, le démarrage de la saison des feux a été progressif depuis le mois d'octobre pour atteindre son pic en février. Une baisse des occurrences de l'ordre de 3\% par rapport à la moyenne est observée. On note également une baisse significative pendant les mois de janvier et avril (Figure 7a). 


\section{Les pays du groupe 3}

$\mathrm{Au}$ Nigeria, le nombre d'occurrences des feux a été similaire à celui de la période 2014-2018. Le cumul des occurrences de cette saison est en hausse de 6\% par rapport à la moyenne des 5 dernières années. La saison des feux commence en octobre. Les plus importantes détections sont observées durant les mois de décembre, janvier et février avec le pic situé en janvier. La comparaison de la variation mensuelle des détections indique que les mois d'octobre, novembre, décembre et janvier ont enregistré moins de feux par rapport à la moyenne de la série. Par contre, le mois de février a enregistré $75 \%$ de plus que la moyenne des 5 dernières années dudit mois (Figure 7b). En Côte d'Ivoire, la saison 2019/2020 a démarré timidement en novembre pour connaître son pic en février. Le cumul des occurrences des feux est en baisse de 13\% par rapport à la moyenne des 5 dernières années. L'analyse de la variation mensuelle montre que les mois de décembre et janvier qui enregistrent en moyenne, la moitié des détections de la saison ont recueilli moins de foyers de feux actifs. En revanche, le mois de février a enregistré 55\% plus de feux que la moyenne des 5 dernières années (Figure 7c).

\section{Les pays du groupe 4}

$\mathrm{Au}$ Togo, le cumul du nombre d'occurrences est de $12 \%$ inférieur à celui de la moyenne des 5 dernières années. La saison des feux a démarré en novembre pour atteindre son maximum en janvier, mais la détection a été pratiquement stable pendant les mois de décembre et janvier. L'analyse comparative des variations mensuelles des foyers de feux actifs montre que le mois de février a enregistré plus de feux. Cependant, les mois de novembre et décembre ont enregistré moins de feux que la moyenne des 5 dernières années (Figure 7d). Par contre, au Ghana, le cumul du nombre d'occurrences des feux actifs au cours de cette saison est en hausse de 3\% par rapport la moyenne de référence. La saison des feux démarre en moyenne en novembre pour connaitre son maximum en janvier, suivi d'une baisse progressive jusqu'en avril. Les plus importantes détections sont enregistrées au cours des mois de décembre et janvier. La variation mensuelle des détections de cette saison comparée à la moyenne indique que le pays a enregistré plus de feux durant les mois de janvier et février (Figure 8a). Quant au Bénin, il y a eu $13 \%$ moins de feux durant la saison 2019/2020 comparé à la moyenne des 5 dernières années. La saison d'occurrences des feux a démarré en novembre 2019 pour se terminer en avril 2020. Les 30\% des détections ont été enregistrés pendant le mois de février. La comparaison des variations mensuelles des détections indique qu'il y a eu moins de feux durant les mois de novembre, décembre et janvier. En revanche, les mois de février, mars et avril ont enregistré plus de feux que la moyenne (Figure 8b).

Les pays du groupe 5

Au Sénégal, le nombre d'occurrences des feux a été de $10 \%$ supérieur à celui de la moyenne de la série de référence. La saison des feux a démarré en octobre. Les plus importantes détections sont faites en novembre, décembre, janvier et février avec un pic en février. La comparaison des variations mensuelles par rapport à la moyenne des 5 dernières années indique qu'il y a eu moins de feux durant les mois de janvier et mars. Par contre, l'on a enregistré plus de feux pendant les autres mois dont $35 \%$ de plus que la moyenne des 5 dernières années en février (Figure 8c). En Mauritanie, c'est une augmentation de $11 \%$ par rapport à la moyenne des 5 dernières années qui a été enregistrée. La saison de feux démarre timidement en septembre pour atteindre son pic en décembre. La comparaison des variations mensuelles des occurrences de feux indique qu'il y a eu plus de feux durant les mois d'octobre, décembre et janvier pendant que les autres mois enregistrent une baisse sensible (Figure 8d).

Au Mali, le cumul total du nombre d'occurrences des feux est en hausse de 7\% par rapport à la moyenne des 5 dernières années. La saison des feux démarre en octobre pour atteindre rapidement son maximum en novembre, suivi d'une baisse progressive jusqu'en avril. La variation mensuelle des détections par rapport à la série de référence indique que les mois d'octobre, novembre, décembre, février et mars ont enregistré plus de 
feux. Les mois de janvier et avril ont enregistré respectivement $25 \%$ et $10 \%$ moins de feu par rapport à la moyenne de la série de référence (Figure 9a).

\section{Les pays du groupe 6}

Au Tchad, on note une hausse $7 \%$ du nombre d'occurrences des feux par rapport à la moyenne de la série de référence. La saison des feux démarre en octobre pour atteindre son pic en décembre suivi d'une baisse progressive des détections jusqu'à la fin avril avec 655 foyers détectés. On peut noter également que, le mois d'octobre a exceptionnelement enregistré moins de feux que la moyenne de la serie de reférence (Figure 9b).

$\mathrm{Au}$ Burkina Faso, le nombre d'occurrences a baissé de $8 \%$. La saison des feux a démarré en septembre pour atteindre son pic en décembre. La comparaison des variations mensuelles des détections indique qu'il y a respectivement $77 \%, 12 \%, 21 \%$ et $12 \%$ moins de feux en octobre, novembre, janvier et mars contre respectivement $3 \%$ et $18 \%$ plus de feux détectés en décembre et février (Figure 9c).

\section{Le pays du groupe 7}

Au Niger, le cumul du nombre d'occurrences des feux actifs a augmenté de $28 \%$ par rapport à la moyenne de référence . La saison des feux a démarré en octobre pour atteindre rapidement son pic en novembre suivi d'une régression progressive jusqu'en avril. Le pic qui se situe en novembre a recueilli $38 \%$ des détections du total annuel. La variation des détections de la saison 2019/2020 comparée à la moyenne des 5 dernières années indique que le Niger a enregistré exceptionnellement plus de feux sur toute la période allant de novembre à avril (Figure 9d).

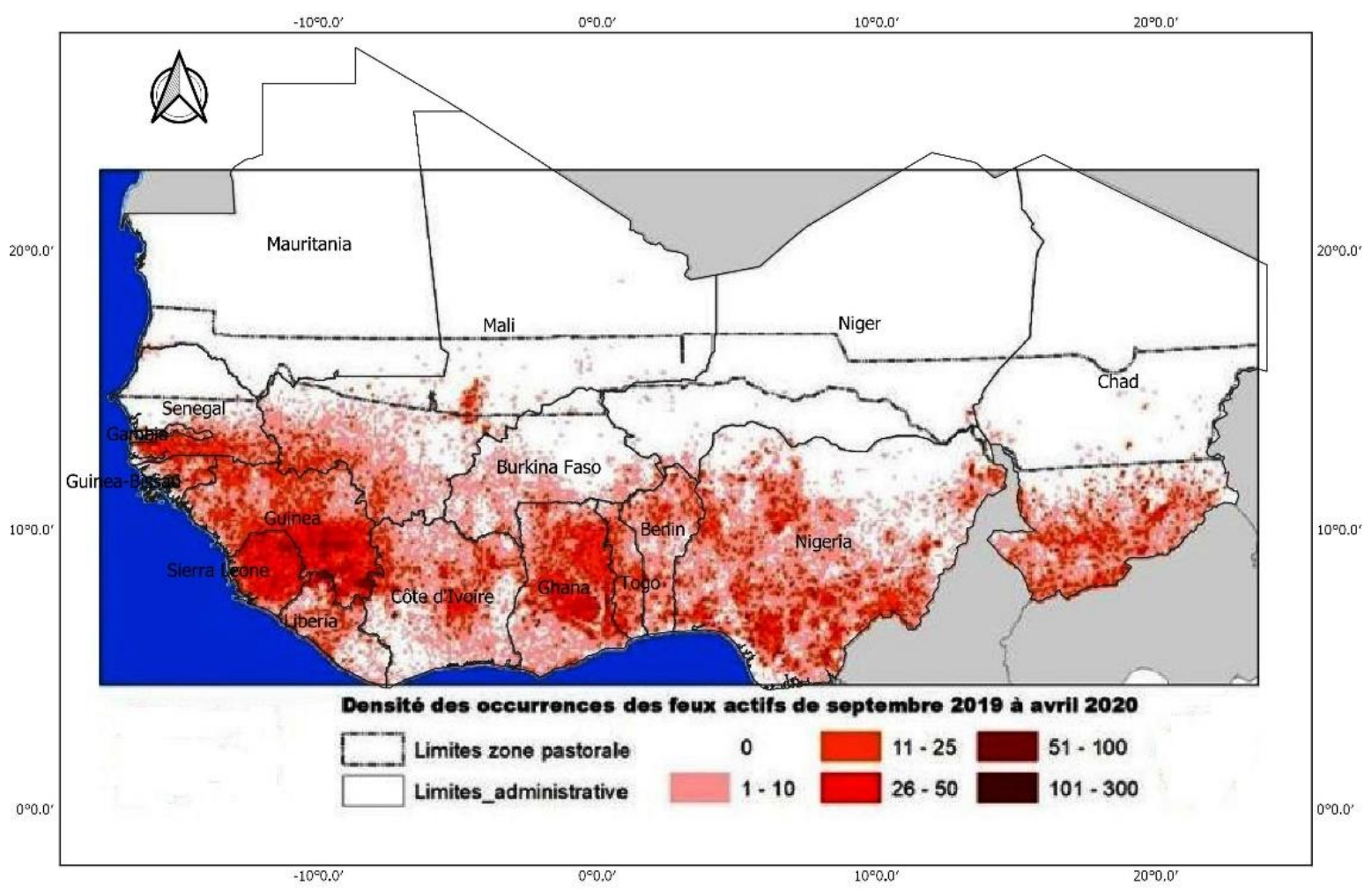

Figure 3 : Densité des occurrences des feux actifs pour la saison 2019/2020 en Afrique de l'Ouest et au sahel. 


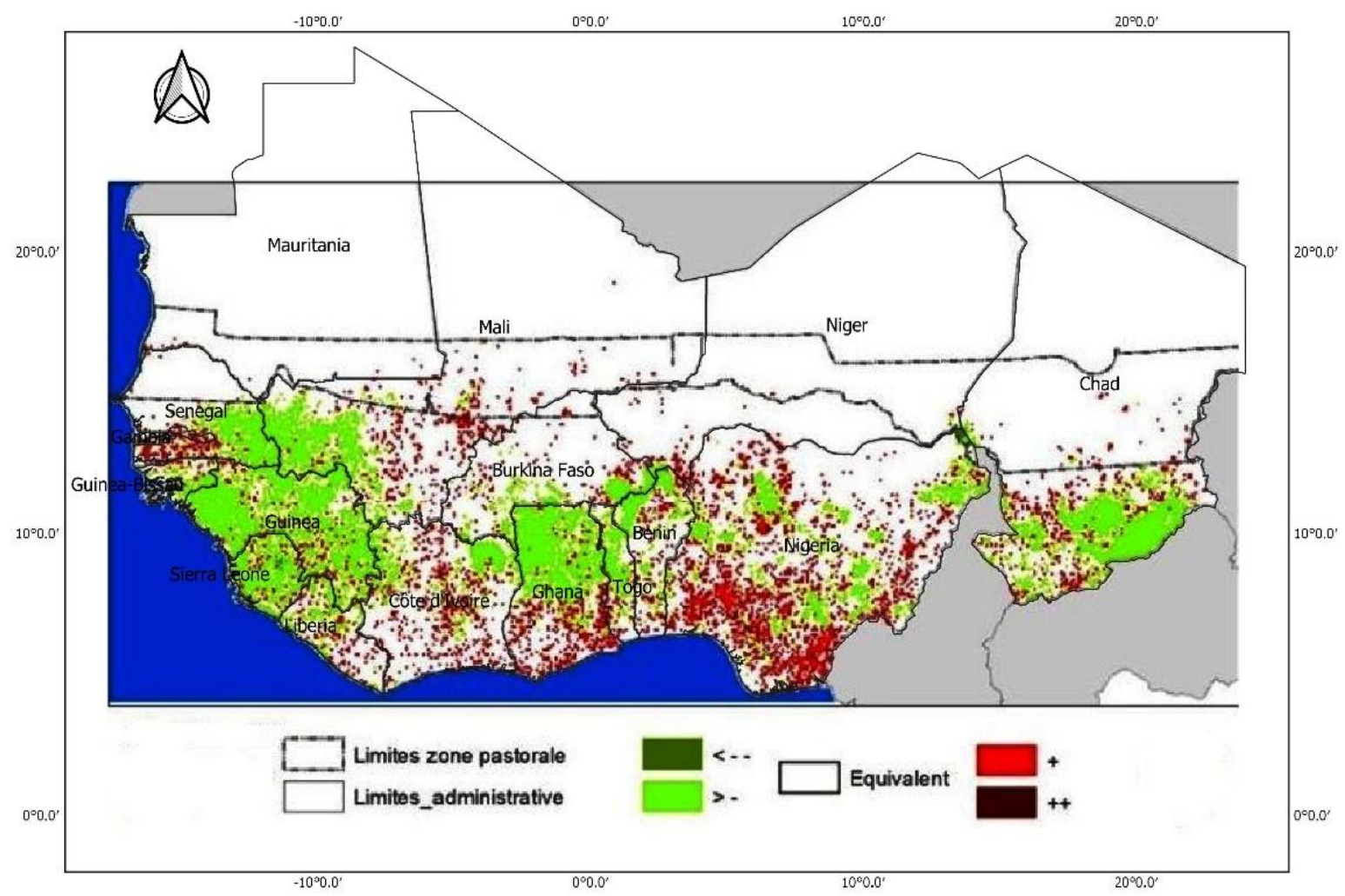

Figure 4 : Comparaison des occurrences des feux actifs pour la saison 2019/2020 à la moyenne des cinq dernières années en Afrique de l'Ouest et au sahel.

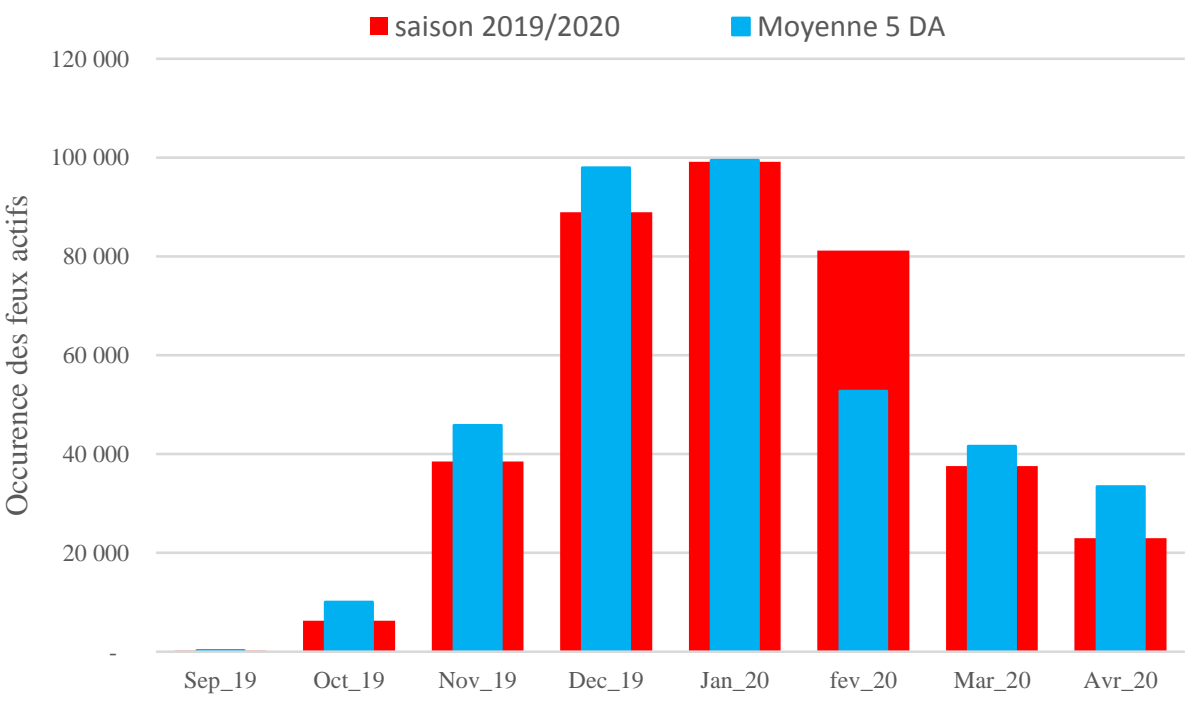

Figure 5 : Variation mensuelle des détections de feux actifs comparée à celle de la moyenne des cinq dernières années sur l'ensemble des pays de l'Afrique de l'Ouest et du sahel. 



Figure 6 : Variation mensuelle des détections de feux actifs comparée à celle de la moyenne des cinq dernières années au : a- Sierra Leone, b- Libéria, c- Gambie, d- Guinée Conakry. 


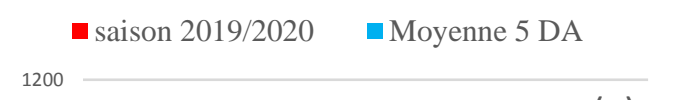

(a)

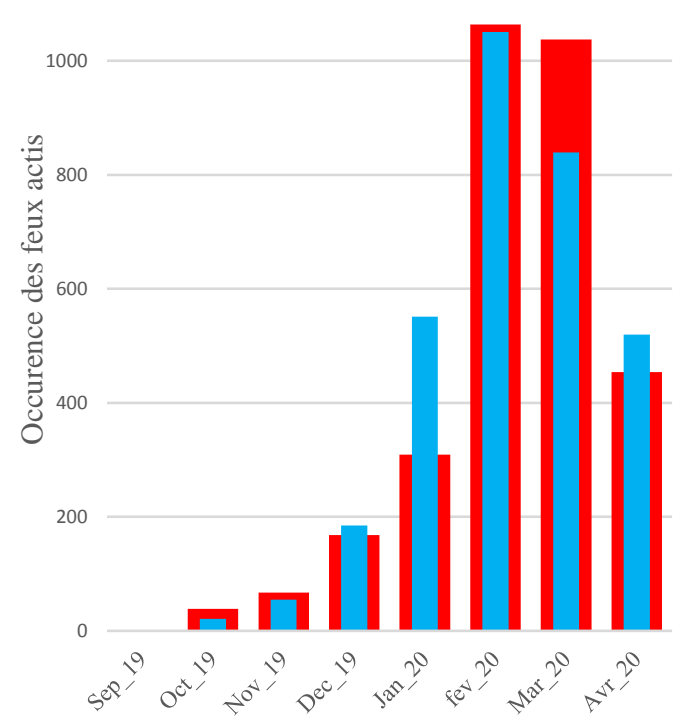

匹 saison 2019/2020 — Moyenne 5 DA

12000

(c)

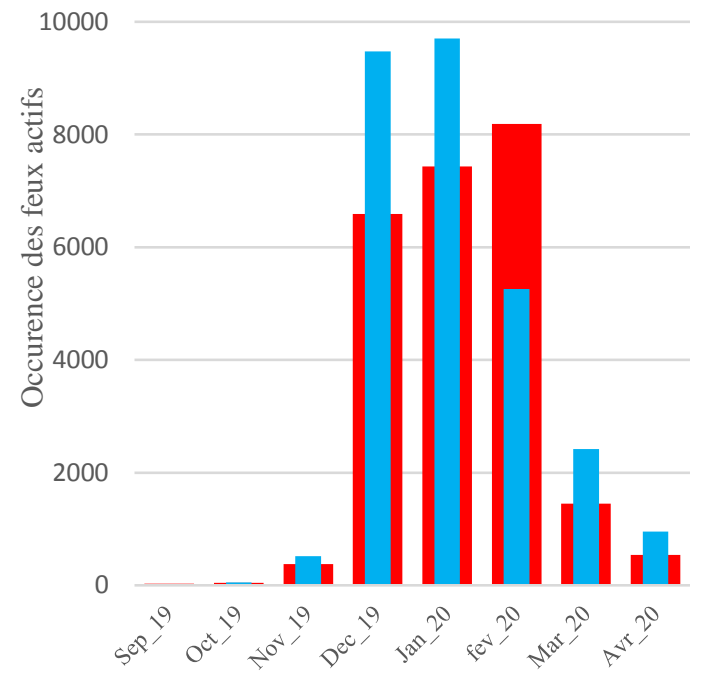

— saison 2019/2020 Moyenne 5 DA

30000

(b)

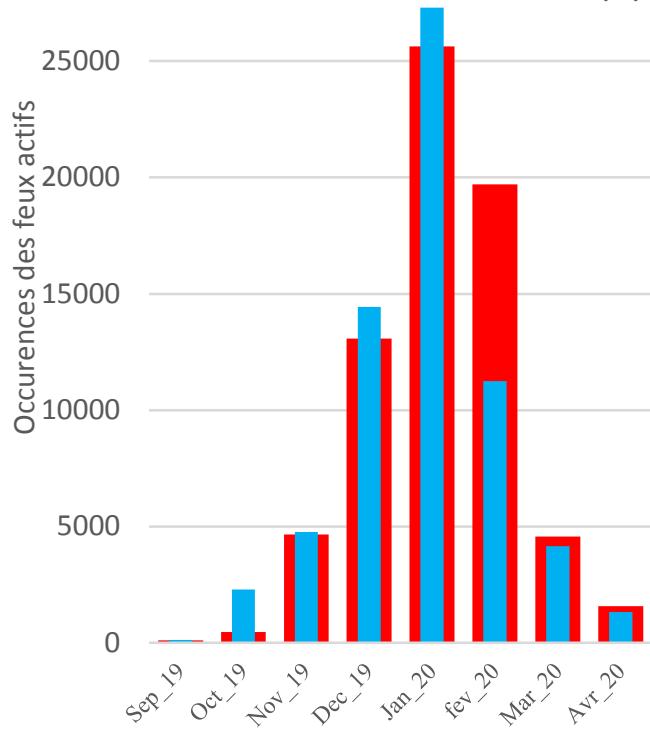

— saison 2019/2020 — Moyenne 5 DA

(d)

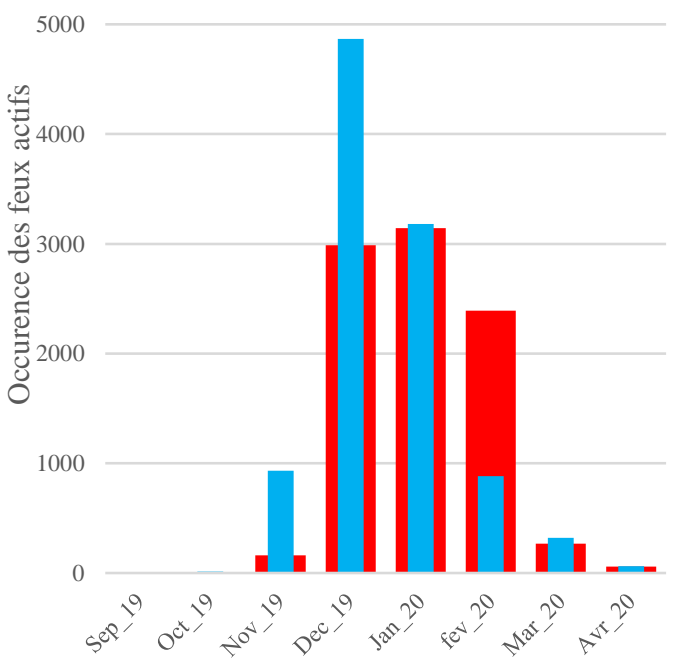

Figure 7: Variation mensuelle des détections de feux actifs comparée à celle de la moyenne des cinq dernières années en : a- Guinée Bissau ; b- Nigeria ; c- Côte d'Ivoire ; d- Togo. 


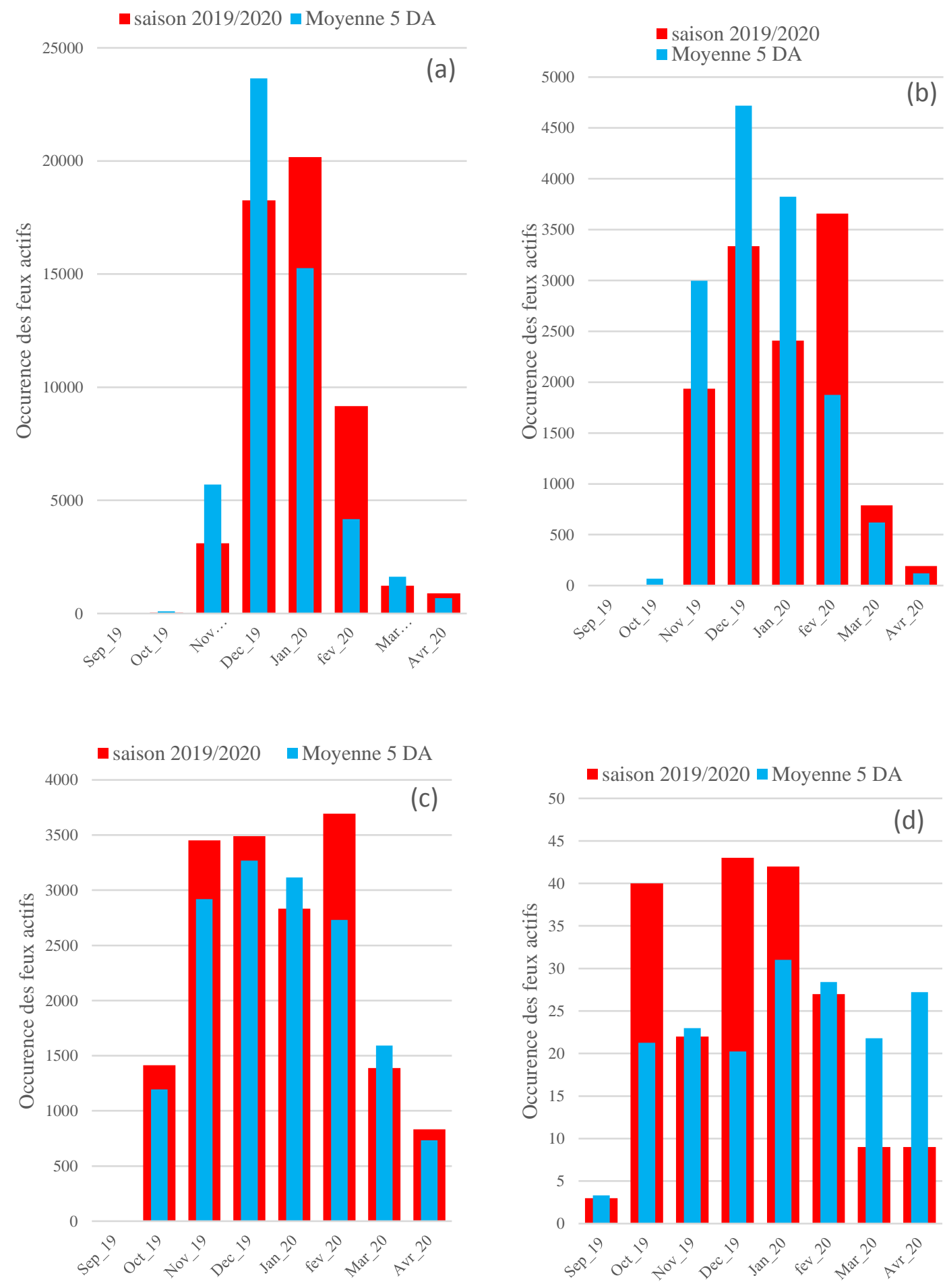

Figure 8: Variation mensuelle des détections de feux actifs comparée à celle de la moyenne des cinq dernières années au : a- Ghana ; b- Bénin ; c- Sénégal ; d- Mauritanie. 

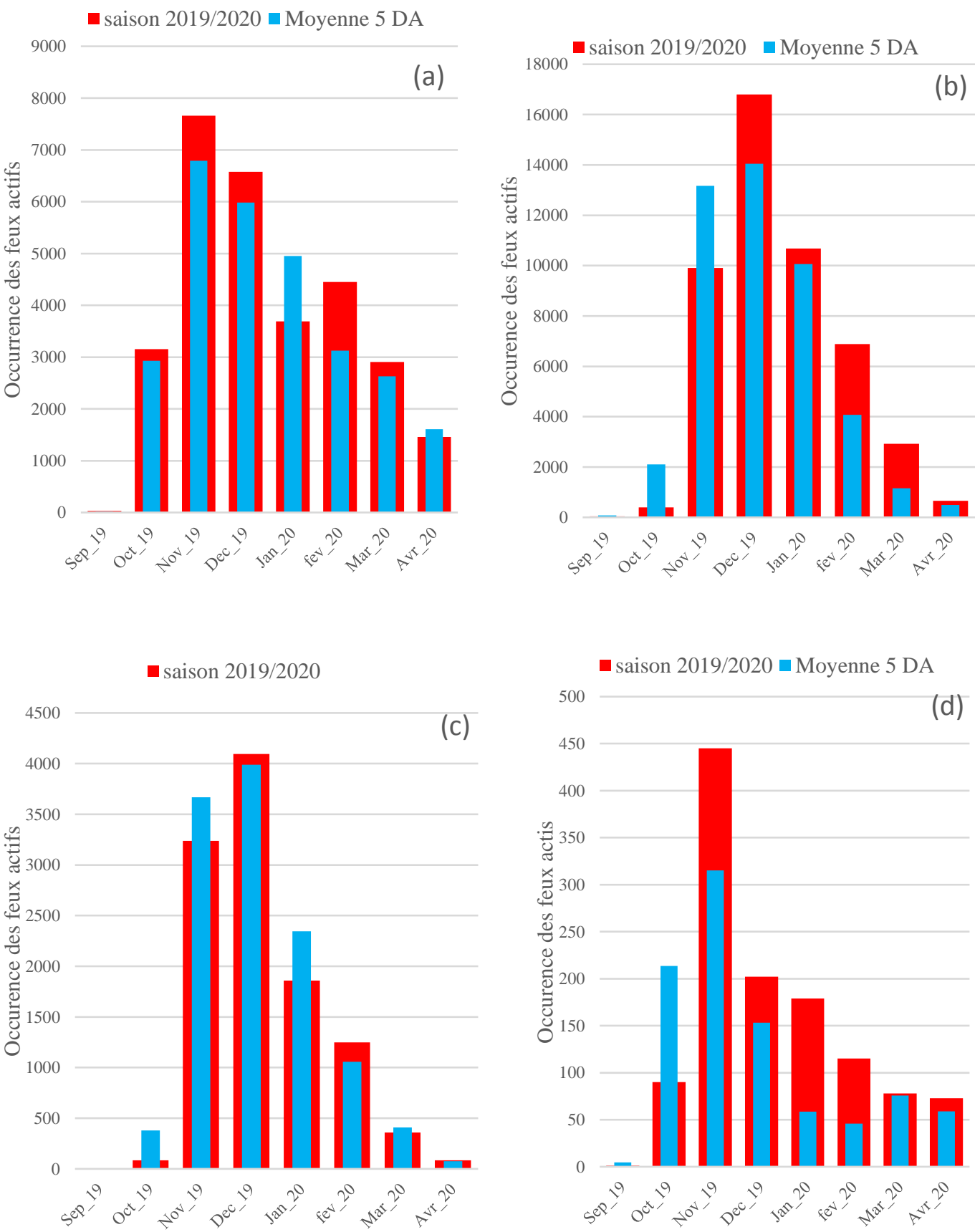

Figure 9: Variation mensuelle des détections de feux actifs comparée à celle de la moyenne des cinq dernières années au : a- Mali ; b- Tchad ; c- Burkina Faso ; d- Niger.DA= Dernières années. 


\section{DISCUSSION}

A l'exception du Burkina Faso, tous les pays du front sahélien ont enregistré plus de feux (sur la saison 2019-2020) par rapport à la moyenne des 5 dernières saisons. Cette situation pourrait s'expliquer par le manque de contrôle dû au contexte sécuritaire, aggravé par les mesures du confinement liées à la pandémie de la COVID-19 mais aussi aux conditions climatiques notamment la baisse du taux d'humidité, les fortes températures ainsi que la vitesse du vent. L'exception que constitue le Burkina Faso est probablement liée à une diminution des activités agricoles parce que cette dernière est aussi à l'origine de certains feux de brousse. En effet, les statistiques du ministère en charge de l'agriculture du Burkina Faso indiquent que les superficies emblavées en 2019/2020 sont en baisse par rapport à la moyenne des 5 dernières années d'au moins $4 \%$ pour le mil, $5 \%$ pour le fonio et $8 \%$ pour le coton. Ces tendances quoique faibles, peuvent expliquer la baisse de détection des feux en 2019/2020 au Burkina Faso. Au Niger, la région la plus soumise au feu est la zone du parc de $\mathrm{W}$ et ses alentours, probablement du fait des brulis effectués pour la mise en culture des terres. En effet la modification de l'occupation des sols dans la Réserve Partielle de Faune de Dosso a été engendrée en substance, par une mise en culture croissante de la brousse tigrée ces dernières années (Idrissa et al., 2019).

Quant aux pays côtiers, la tendance générale des occurrences des feux est à la baisse par rapport à la moyenne des occurrences des 5 dernières années à l'exception de la Gambie où une hausse importante a été observée. Cependant, dans d'autres pays, le nombre d'occurrence des feux est légèrement à la hausse par rapport à la moyenne des 5 dernières années. C'est notamment le cas du Sénégal, du Nigéria et du Ghana. Pour le Nigéria, la hausse de détection des occurrences des feux pour la saison 2019/2020 peut être liée à la zone du Delta du Niger, une zone moins contrôlée à cause de la résurgence en 2018, de l'insécurité liée aux activités pétrolières. En analysant la Figure 3, il peut être loisible d'attribuer la hausse de détection des occurrences des feux au Sénégal à la situation d'insécurité qui prévaut en Casamance, ce qui contribue à compromettre le contrôle des feux dans cette région. Cette situation a probablement influencé la hausse des occurrences des feux en Gambie du fait de l'étroite proximité des deux zones. Quant aux pics des feux au Burkina, les résultats de détection sont corroborés par ceux de Devineau et al. (2010) qui ont situé le pic des feux en novembre et décembre.

On remarque également que les pays ayant une composante forestière enregistrent des occurrences répétitives de feu par rapport aux pays à composantes sahéliennes probablement à cause de la disponibilité de la biomasse. La présence continue d'un tapis herbacé sec favorise le développement des feux de brousse. Au niveau des pays à composante sahélienne bien que la température soit élevée, avec un faible taux d'humidité, la rareté de la biomasse conduit à une diminution des occurrences des feux de brousse comparativement aux pays à composante forestière.

En effet, dans une étude réalisée dans la partie sahélienne et soudanienne du Sénégal, Sow (2013) conclut que la probabilité du mécanisme de mise à feu est influencée par le taux d'humidité de la biomasse et l'humidité relative de l'air. Dans une autre étude réalisée en Argentine, Fischer (2011) conclut également que les variations spatiale et temporelle des feux de brousse dépendent entre autres de la présence d'agriculture, ceci est en accord avec les conclusions de la présente étude. Au Cameroun, Kana et Etouna (2006) avaient conclu que $36 \%$ des savanes arbustives, $23 \%$ des savanes boisées, $7 \%$ des forêts dégradées et seulement $0,4 \%$ des forêts denses humides ont brulé durant leur période d'étude. Christelle et al. (2018) avaient également conclu que les feux de brousse concernent essentiellement les savanes, les forêts sont peu touchées. En effet, la présence continue du combustible (biomasse), avec un taux d'humidité relativement faible durant la période novembre-avril constituent quelquesunes des causes des feux de brousse. 


\section{Conclusion}

Cette étude nous a montré l'importance de la télédétection pour le suivi des feux de brousse. L'analyse des occurrences des feux de la saison 2019/2020 faite sur les données obtenues à travers MODIS a montré que la saison des feux démarre à partir du mois d'octobre et prend fin en avril. Une baisse de détection des feux de l'ordre de $2 \%$ est enregistrée dans les pays de l'Afrique de l'Ouest et du Sahel comparé aux 5 dernières années. Le pic de détection des feux commence à partir du mois de décembre pour la plupart des pays. La répartition temporelle des feux indique que $70 \%$ des détections sont enregistrées entre les mois de décembre et février. Toutes ces informations réunies auxquelles s'ajoutent la connaissance des environnements les plus exposés aux feux, permettront aux décideurs ainsi qu'aux agriculteurs et éleveurs de comprendre le phénomène des feux de brousse dans sa globalité afin de mieux l'encadrer.

\section{CONFLIT D'INTERETS}

Les auteurs déclarent qu'il n'existe aucun conflit d'intérêts.

\section{CONTRIBUTIONS DES AUTEURS}

IG a initié cet article et a procédé au traitement des données et à la production de la première mouture. SAA a effectué l'analyse des données, la recherche bibliographique et l'amélioration de cet article. BB et SO ont examiné cet article et apporté des conseils techniques.

\section{REMERCIEMENTS}

Les auteurs remercient le centre régional AGRHYMET, structure dans laquelle cette étude a été réalisée.

\section{REFERENCES}

Ahouangan BD, Houinato M, Ahamide B, Agbossou E, Sinsin B. 2010. Etude comparative de la productivité de repousses et de la capacité de charge des hémicryptophytes soumises aux feux de végétation dans les parcelles irriguées et non irriguées dans la Réserve Transfrontalière de Biosphère ( RTB ) du
W - Benin. Int. J. Biol. Chem. Sci., 4(2): 479-490. DOI: 10.4314/ijbcs.v4i2.58157

Christelle DG, Martin T, Corneille RF. 2018. Dynamique spatio-temporelle des feux de brousse dans le Parc National du Nbam et Djerem (Camerou). Int. J. Biol. Chem. Sci., 12(2): 728-748. DOI: https://dx.doi.org/10.4314/ijbcs.v12i2.10

Cisse A, Ouattara M, N'Guessan EA, Abrou JE. 2020. Diversité végétale et usages des plantes dans une zone de savane soudanienne: Cas de la localité de Ferkessédougou (Nord, Côte d'Ivoire). Int. J. Biol. Chem. Sci., 14(8): $2807-$ 2825.

DOI:

https://doi.org/https//dx.doi.org/10.4314/ ijbcs.v14i8.13

Devineau J, Fournier A, Nignan S. 2010. Savanna fire regimes assessment with MODIS fire data: Their relationship to land cover and plant species distribution in western Burkina Faso (West Africa). $J$. Arid Environ., 74: 1092-1101. DOI: https://doi.org/10.1016/j.jaridenv.2010.0 3.009

Doamba SWMF, Savadogo P, Nacro HB. 2014. Rôle des feux de savane sur les caractéristiques biogéochimiques des sols en zone soudanienne du Burkina Faso. Int. J. Biol. Chem. Sci., 8(2): 777-793. DOI: https://doi.org/10.4314/ijbcs.v8i2.34

Fischer MA, Bella CMD, Jobbagy EG. 2011. Fire patterns in central semiarid Agentina. J. Arid Environ., 78: 161-168. DOI: https://doi.org/10.1016/j.jaridenv.2011.1 1.009

GIEC. 2014. Changements climatiques 2014 : Incidence, adaptation et vulnérabilitéRésumé à l'intention des décideurs. Contribution du groupe de travail II au cinquième rapport d'évaluation du groupe d'experts intergouvernemental sur l'évolution du climat, Organisation météorologique mondiale, Genève (Suisse), 34p.

Hiernaux P, Le Houérou HN. 2006. Les parcours du Sahel. Secheresse, 17: 51-71.

Idrissa KA, Tougiani A, Moussa M, Habou R, Idrissa S, Jan B. 2019. Influence des pressions anthropiques sur la dynamique 
paysagère de la Reserve Partielle de Faune de Dosso (Niger). Int. J. Biol. Chem. Sci., 13(2): 1094-1108. DOI: https://dx.doi.org/10.4314/ijbcs.v13i2.41

Kana CE, Etouna JE. 2006. Apport de trois méthodes de détection des surfaces brûlées par imagerie Landsat ETM+: application au contact forêt- savane du Cameroun. Eur. J. Geography. DOI: https://doi.org/https://doi.org/10.4000/cy bergeo. 2711

Kganyago M, Shikwambana L. 2020. Assessment of the Characteristics of Recent Major Wildfires in the USA, Australia and Brazil in 2018-2019 Using Multi-Source Satellite Products. Remote Sens., 12: $1803 . \quad$ DOI: https://doi.org/10.3390/rs12111803

Le TH, Nguyen TNT, Lasko K, Ilavajhala S, Vadrevu KP, Justice C. 2014. Vegetation fires and air pollution in Vietnam. Environ. Pollut., 195: 267-275. DOI: https://doi.org/10.1016/j.envpol.2014.07. 023

Lin HW, Jin Y, Giglio L, Foley JA, Randerson JT. 2012. Evaluating greenhouse gas emissions inventories for agricultural burning using satellite observations of active fires. Ecol. Apllications, 22: 13451364. DOI: https://doi.org/10.1890/102362.1

N'Dri AB, Fongbe M, Soro TD, Gignoux J, Kone M, Dosso K, N'Dri JK, Kone NA, Barot S. 2018. Principaux indices de l'intensité du feu dans une savane Guinéenne d'Afrique de l'Ouest. Int. J. Biol. Chem. Sci., 12(1): 266-274. DOI: https://doi.org/https://dx.doi.org/10.4314 /ijbcs.v12i1.21

Price OF, Williamson GJ, Henderson SB, Johnston F, Bowman DMJS. 2012. The relationship between particulate pollution levels in australian cities, meteorology, and landscape fire activity detected from MODIS hotspots. PLoS One, 7(10): e47327.

DOI: https://doi.org/10.1371/journal.pone.004
7327

Sow M, Hély C, Mbow C, Sambou B. 2013. Fuel and fire behavior analysis for earlyseason prescribed fire planning in Sudanian and Sahelian savannas. J. Arid Environ., 89: 84-93. DOI: https://doi.org/10.1016/j.jaridenv.2012.0 9.007

Takahata C, Amin R, Sarma P, Banerjee G, Oliver W, Fa JE. 2010. Remotely-Sensed Active Fire Data for Protected Area Management : Eight-Year Patterns in the Manas National Parks, India. Environ. Manage., 45: 414-423. DOI: https://doi.org/10.1007/s00267-0099411-8

Valea F, Ballouche A. 2012. Les feux de brousse en Afrique de l'Ouest: contraintes environnementales ou outil de gestion environnementale? L'exemple du Burkina Faso. Territ. d'Afrique, 3: 3647.

URL https://www.researchgate.net/publication /257298202

Wang Y, Huang J, Zananski TJ, Hopke Pk, Holsen TM. 2010. Impacts of the Canadian Forest Fires on Atmospheric Mercury and Carbonaceous Particles in Northern New York. Environ. Sci. Technol, 44: 8435-8440. DOI: https://doi.org/10.1021/es1024806

Zeineddine N. 2020. La reprise des pluies et la recrudescence des inondations en Afrique de l'Ouest sahélienne. Physio-Géo., 89109.

DOI:

https://doi.org/10.4000/physiogeo.10966

Zhang J, Yao F, Liu C, Yang L, Boken VK. 2011. Detection, emission estimation and risk prediction of forest fires in China using satellite sensors and simulation models in the past three decades-An overview. Int. J. Environ. Res. Public Health, 8: 3156-3178. DOI: https://doi.org/10.3390/ijerph8083156 